\title{
CONTROLE ESTATÍSTICO DE PROCESSOS PARA ANÁLISE DE DEVOLUÇÕES: ESTUDO EM UMA DISTRIBUIDORA DE FRIOS
}

\author{
Emanoel Henrique Alves Medeiros (INSTITUTO FEDERAL DO ESPÍRITO SANTO) \\ emanoel.medeiros.log@ gmail.com \\ Paulo Henrique dos Santos (INSTITUTO FEDERAL DO ESPÍRITO SANTO) \\ paulo.santos@ifes.edu.br
}

\section{Resumo}

O objetivo deste artigo é estabelecer Controle Estatístico de Processos (CEP) sobre o índice de devoluções gerado em uma transportadora de frios no período de 121 dias. O método utilizado, foi estudo de caso de caráter qualitativo e quantitativo. Houve aplicação de ferramentas CEP, tais como, "gráfico de controle por atributo", "gráfico de Pareto", "diagrama de causa e efeito", do mesmo modo, ferramentas da qualidade, tais como, "5W2H" e "brainstorming" com objetivo de acompanhar a variabilidade dos eventos e mensurar os impactos causados pelos mesmos, além de estabelecer um controle e propor melhorias no processo. O estudo foi desenvolvido através de uma investigação empírica, com foco no controle de processos, qualidade e redução de variabilidades operacionais.

Palavras-Chaves: Controle estatístico de processos, Qualidade, Devoluções.

\section{Introdução}

Os clientes finais estão cada vez mais exigentes devido à variedade de oferta do mercado, para atender a demanda com eficiência fornecedores vem buscando vantagens competitivas, Camarotto (2009). O autor ressalta que uma empresa que comercializa produtos deve estar atenta aos concorrentes focando oferecer serviço logístico de qualidade para obter vantagens competitivas sobre estes concorrentes. Operações logísticas impactam diretamente na eficiência do fornecedor que terceiriza o serviço logístico e foca em sua principal atividade, o comercio de produtos (CHOPRA; MEINDL, 2007).

Empresas terceirizadas optam em utilizar ferramentas de controle para auxiliar na gestão de seus processos e atender o nível de serviço estipulado pelo fornecedor contratante além de profissionais qualificados que saibam analisar e tomar a melhor decisão para a resolução de problemas com o menor custo possível (WANKE, 2004). 
Usando como escopo a distribuição, uma empresa terceirizada que atua como Operador Logístico 3PL no seguimento de frios e congelados a qual utiliza como um dos principais indicadores de qualidade no serviço o índice de devolução. Neste cenário, justifica-se desta a implantação do (CEP), observando a operação empírica. Segundo Lachman et al. (2001) através deste controle é possível distinguir resultados especiais de resultados normais, permitindo medir a variação de determinado valor dentro de um lote de fabricação ou dentro de um processo analisado.

Grande dificuldade das empresas é encontrar gestores qualificados que saibam tomar decisões após analisar relatório, não basta ter dados históricos é preciso ter gestão de conhecimento e propor melhor resolução em tratativas de anomalias (PROBST; RAUB; ROMHARDT, 2002).

Como problemática, pretende-se responder as seguintes questões: quais devoluções têm a maior representatividade dentro dos dados analisados? Quais as tratativas necessárias para cada tipo de devolução? Quais soluções propor para estabilizar as anomalias no processo?

O objetivo deste artigo é analisar as devoluções na fase de distribuição da empresa estudada, descrevendo suas respectivas representatividades, tratamentos específicos por devolução e propondo melhorias baseadas nos resultados das ferramentas da qualidade utilizadas.

Este artigo está estruturado da seguinte maneira, primeira seção a introdução, segunda seção o contexto teórico aplicado no estudo, em seguida a terceira seção contendo os procedimentos metodológicos, a quarta seção a análise e discussão dos resultados, por fim, a seção de conclusão, respondendo o objetivo do estudo, suas contribuições, limitações e propondo pesquisas futuras.

\section{Referencial teórico}

\subsection{Cartas de controle}

No sistema de controle estatístico é aceitável e normal a variabilidade, desde que esteja dentro do controle estipulado pela ferramenta. O gráfico compõe-se por três linhas, contendo uma linha na horizontal, denominada de linha de centro (LC), que representa o valor médio ou ponto de controle aceitável, outras duas linhas, também na horizontal que são o limite superior controlado (LSC) e o limite inferior controlado (LIC) (MARTINS; LAUGENI, 2005). identifica-se desvios padrões que variam a partir de uma linha de centro, avaliando se o 
processo está sobre controle e se todos os pontos amostrais estão entre o (LI) e o (LS) conforme demostrado na Figura 1.

Figura 1 - Gráfico de controle.

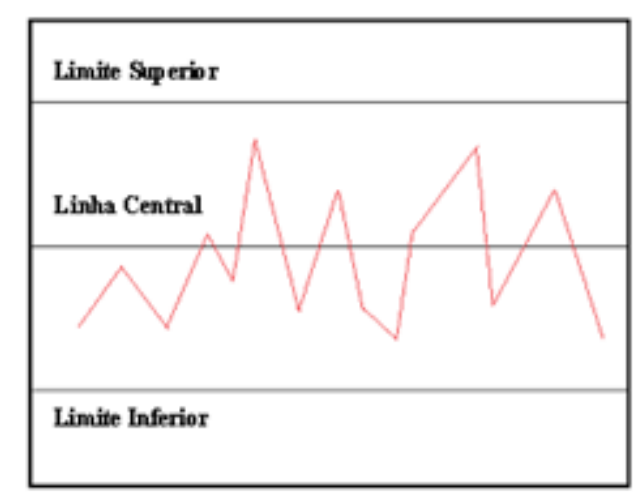

Fonte: Montgomery (2019, p.131).

Segundo Andrade (2011) existem dois tipos de gráficos de controle, por variáveis ou por atributos. No gráfico por variáveis o objetivo e controlar a variabilidade do processo e mensurar numericamente de forma quantitativa esta variabilidade. Muitas características do processo de qualidade não podem ser medidas em uma escala quantitativa ou contínua, para a mensuração deste controle é utilizado o gráfico de controle por atributos.

Existem três tipos de controle por atributos, são eles: gráfico de controle para fração não conforme (p), gráfico de controle para não conformidades (c) e gráfico de controle para não conformidades de unidades (u) (MONTGOMERY, 2019). Considerando o objetivo deste estudo que é analisar as devoluções de uma transportadora, utiliza-se o gráfico de controle para não conformidades (c).

\subsection{Diagrama de Pareto}

O gráfico de Pareto foi criado por Vilfredo Pareto com objetivo de medir a desigualdade social através da distribuição da renda da Itália, através deste estudo foi possível identificar que $80 \%$ de toda riqueza estava concentrada nas mãos da pequena parcela da população e os outros 20\% estavam concentrados na maioria da população, o gráfico também ficou conhecido com gráfico 80/20 segundo (PALADINI, 2012).

O gráfico organiza as classificações da base de dados em ordem decrescente da esquerda para a direita em forma de barras, auxiliando na análise e demostrando que a maioria dos defeitos representam poucas causas (SELEME; HUMBERTO, 2012). 
No ambiente empresarial, esta ferramenta auxilia o gestor a reconhecer o acúmulo de ocorrências e situações problemáticas, permitindo o mesmo tomar decisões para cada tipo de ocorrência. Através deste gráfico é possível medir as influências de cada defeito no processo analisado (BEHR, 2008). O acompanhamento da variação destes defeitos favorece o gestor a tomar decisões que objetivam na resolução daquela anomalia e diminui o impacto causado pela mesma (PEINADO; GRAEML, 2007).

\subsection{Diagrama de causa e efeito}

O objetivo da ferramenta é encontrar a raiz dos problemas, buscando entender quais foram os fatores primários que contribuíram para que certa anomalia tenha aparecido. Toledo (2014) destaca que a ferramenta foi desenvolvida para representar de forma gráfica a compreensão lógica dos fatos, facilitando a visualização dos fatores apresentados. $\mathrm{O}$ autor ressalta que na fase de implantação e construção do diagrama deve-se executar uma técnica brainstorming para melhor resolução da anomalia central.

De acordo com Montgomery (2019), após a identificação do defeito, deve-se iniciar a análise das possíveis causas deste defeito, de forma separadamente, o autor ressalta que para a construção do diagrama de causa e efeito, deve-se seguir sete passos, a saber:

- $\quad$ Definição do problema ou defeito analisado;

- Formar equipe para realizar a análise de causas potenciais em seções de brainstorming;

- $\quad$ Desenhe a caixa de efeito e a linha central;

- Especificar as principais categorias de causas potenciais e coloque-as em caixas, ligadas à linha central;

- Identificar as causas possíveis e classificar na categoria do passo anterior e criar categorias se necessário;

- Ordenar as causas para identificar aquelas que parecem mais prováveis de causar impacto sobre o problema;

- $\quad$ Adote ações corretivas. 


\section{Procedimentos metodológicos}

Para obter resultados significantes, Montgomery (2019) apresenta o passo a passo da implantação de um programa de CEP bem-sucedida, o processo de controle estatístico deve ser dividido em seis etapas:

- $\quad$ Liderança gerencial;

- $\quad$ Abordagem de equipe, com foco em aplicações orientadas pelo projeto;

- $\quad$ Educação dos empregados em todos os níveis;

- $\quad$ Enfase na redução de variabilidade;

- $\quad$ Avaliação do sucesso em termos quantitativos (econômicos);

- $\quad$ Mecanismo para comunicar os resultados de sucesso por toda empresa.

Na primeira etapa o gerente deve ter compromisso com a implantação, nesta deve-se decidir quais características controlar, especificando os limites de controle, tipo do gráfico de controle, tomada de ações e utilização de sistemas de computador.

Na segunda etapa de aplicação, é importante reunir a equipe que será responsável em executar a aplicação e fazer uma integração da mesma com a ferramenta CEP. Após esta integração a gerência deve apresentar a área a ser controlada, os dados processados e estipular metas. Com o controle estipulado, inicia-se terceira etapa, aplicada de forma educativa, ofertando um treinamento para todos colaboradores envolvidos no processo de melhoria.

Na quarta etapa, deve-se focar na variabilidade e redução dos eventos não conformes, nesta etapa, sugere-se a necessidade de utilização de outras ferramentas CEP, como o gráfico de Pareto.

Na quinta etapa, deve-se avaliar de forma quantitativa e em termos econômicos o impacto dos eventos não conformes citados no gráfico de Pareto. Uma vez que o evento não conforme tenha sido identificado, ressalta-se a importância de utilização de seções de brainstorming com a equipe focado em atacar a raiz do evento não conforme e definir as principais causas e seus respectivos efeitos, através da aplicação de outra ferramenta CEP o diagrama de Ishikawa.

Na sexta etapa, deve-se criar um mecanismo de comunicação para toda empresa, e apresentar os resultados de sucesso das áreas controladas. 


\subsection{Método utilizado}

O método utilizado no artigo é o estudo de caso, o qual tem como objetivo investigar os dados contemporâneos de determinado padrão ou contexto que não estão bem definidos (YIN, 2001, p. 32). Ressalta-se que o estudo de caso é uma investigação empírica da vivência do investigador. Segundo Collins e Hussey (2005) existem dois paradigmas a ser definidos antes de iniciar o estudo, estipular se a pesquisa é qualitativa ou quantitativa. Neste contexto a pesquisa será considerada tanto qualitativa quanto quantitativa.

André (2005), contextualiza três etapas para implantação de um estudo de caso: fase exploratória que define o foco do estudo, analisar as devoluções na fase de distribuição da empresa estudada, descrevendo suas respectivas representatividades, tratamentos específicos por devolução e propondo melhorias baseadas nos resultados das ferramentas da qualidade utilizadas. Fase de coleta dos dados, apresentados na seção 3.3 intitulada "Tratamento dos dados". Fase de análise sistemática dos dados, apresentados na seção 4 intitulada "Análise e discursão dos resultados".

Após a aplicação do estudo de caso, é possível obter método de pensamento que reflete o tratamento científico e a mensuração de verdades absolutas e parciais. (LAKATOS; MARCONE, 2009).

\subsection{Objeto do estudo}

Fundada em 2003, na cidade de Cariacica, Estado do Espírito Santo, a Gilles logística é conta com um centro de distribuição de (CD) 9.725,86 $\mathrm{m}^{2}$, especializada em transporte, armazenagem e distribuição de produtos alimentícios refrigerados dede área total, localizado na cidade de Cariacica, Espírito Santo. A operação da empresa é dividida em quatro etapas, transferência, expedição, distribuição e prestação de contas. Na Figura 2, será apresentado o fluxo de processos deste setor.

O objetivo do setor de expedição é garantir que a mercadoria seja embarcada da forma certa, com a qualidade desejada e com o menor custo possível. Ao analisar este fluxo é possível constatar que nas ocorrências das devoluções é gerado um empenho do setor para tratar esta anomalia. A tratativa gera hora extra, que é um custo operacional, além de reduzir a produtividade da equipe e reduzir o nível de serviço. 
Figura 2 - Fluxo de processos de expedição.

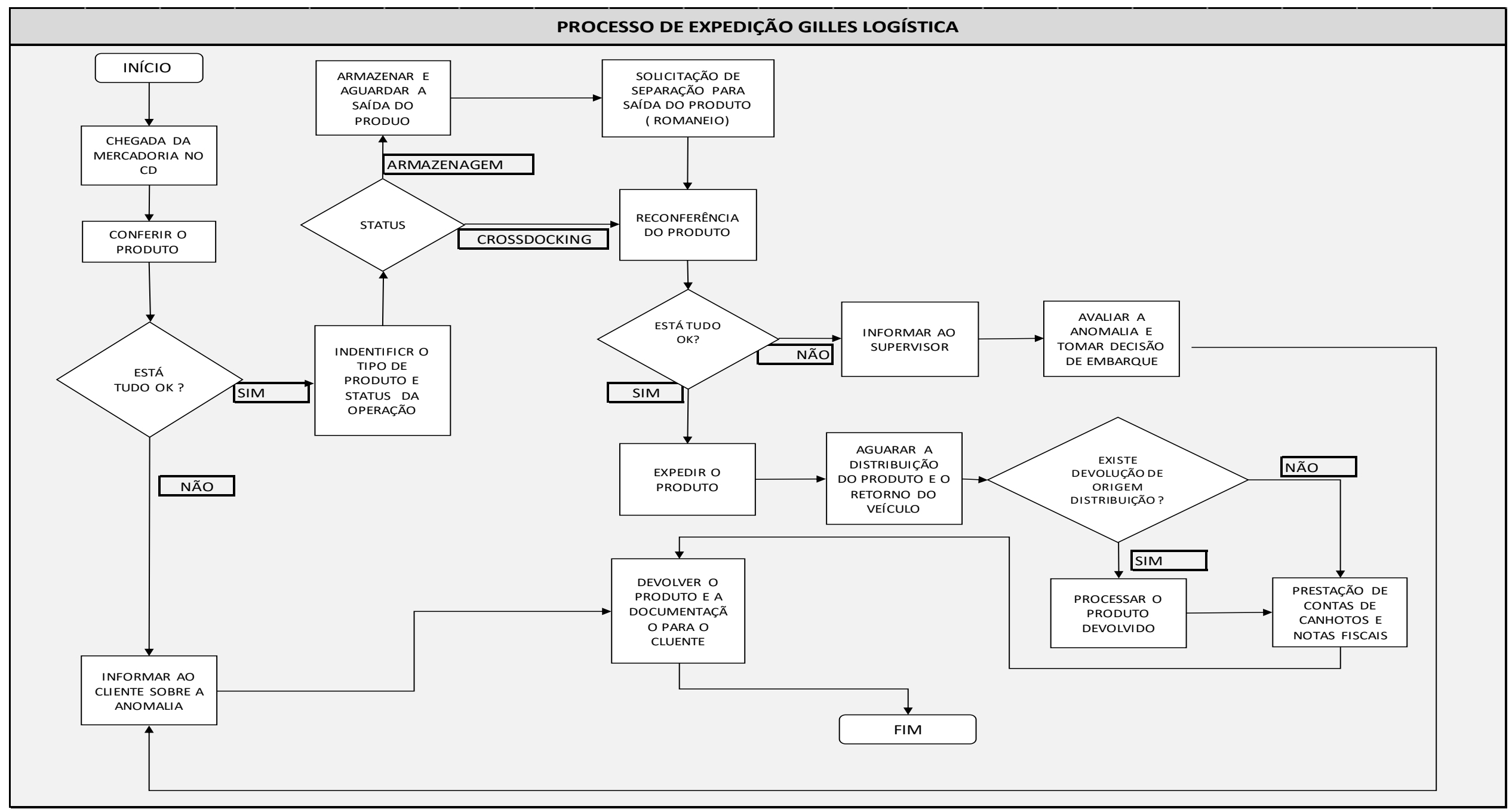

Fonte: Elaborada pelo autor. 


\subsection{Tratamento dos dados}

Utilizando como metodologia o modelo de implantação do CEP, vista em Montgomery (2019), ressaltando que o gerente da empresa tem importância significante para que a implantação do CEP seja bem-sucedida. Na primeira etapa de implantação da ferramenta o gerente extraiu do sistema da empresa relatório de devolução entre (01/01/2019 a 01/07/2019) totalizando 121 observações. Neste relatório continham as seguintes variáveis (Data, Descrição da ocorrência, Peso bruto, Rota, Causador e Observação).

Para auxiliar na análise dados, o gerente utilizou uma ferramenta habilitada no Excel chamada Acition para possibilitar a aplicação da carta de controle tipo (c). Esta ferramenta foi desenvolvida em 2005 por um grupo de doutores em computação e estatística da empresa Estatcamp.

\section{Análise e discussão dos resultados}

Utilizando como auxílio a ferramenta Action, os dados foram coletados, processados foi criado o gráfico de controle para não conformidade (c). O objetivo deste gráfico é identificar quais características do processo devem ser controladas e se o mesmo está sob controle, além de acompanhar a variabilidade dos eventos especiais, conforme demostrado na Figura 3.

Figura 3 - Gráfico c para devoluções.

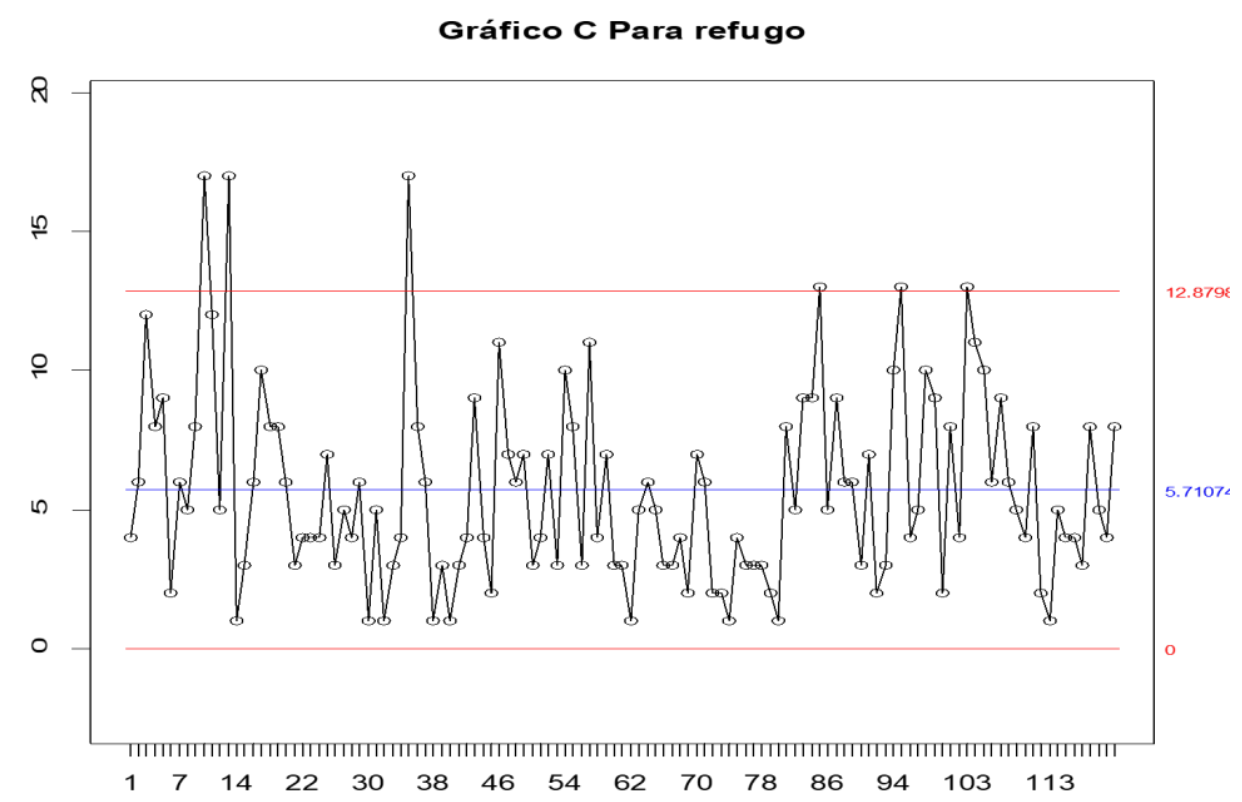

Fonte: Elaborada pelo autor. 
Após aplicar o gráfico, é possível analisar na Figura 3 a variabilidade do índice de devolução durante o período processado, o gráfico traz LS de 12,87 devoluções por dia, M de 5,710 por dia e L.I de 0 devolução por dia.

Na segunda etapa com a definição dos limites de controle, o gerente reuniu uma equipe (Supervisor de Expedição, Encarregada de monitoramento, Encarregado de expedição, Roteirizador e Supervisor Operacional) para demostrar os resultados indicados no gráfico de controle. Após a apresentação dos resultados foi aplicado a terceira etapa, um treinamento da equipe de integração com a ferramenta CEP.

A quarta etapa inicia-se com a equipe treinada e integrada sobre a ferramenta CEP, é feito um Brainstorming, com ênfase na redução da variabilidade. Analisando os dados da Figura 3, a equipe constatou que em 6 dias a quantidade de devolução ultrapassou o LS. Foi explorada e identificado pela equipe, que tratava-se de clientes fechados por motivos particulares dos mesmos, após esta análise e seguindo as diretrizes de Santos et al, (2016) a equipe junto com o gerente constatou que nas causas especiais ouve falhas ocasionadas que ocorrem durante o processo, com grande influência individual ou seja, devoluções causadas especificamente pela impossibilidade de entregar a mercadoria ao cliente, feriados municipais não informados e)ou não verificados antes da consolidação da carga durante o processo de roteirização, clientes fechados por motivos pessoais e calamidades pública. Além disto as causas especiais acusam erros de operação imprecisão no ajuste de processos dentre outras variações. Após esta análise retirou-se estes seis eventos especiais da amostra e foi aplicado um novo gráfico de controle conforme demonstração na Figura 4. 
Figura 4 - Gráfico c para devoluções sem eventos especiais.

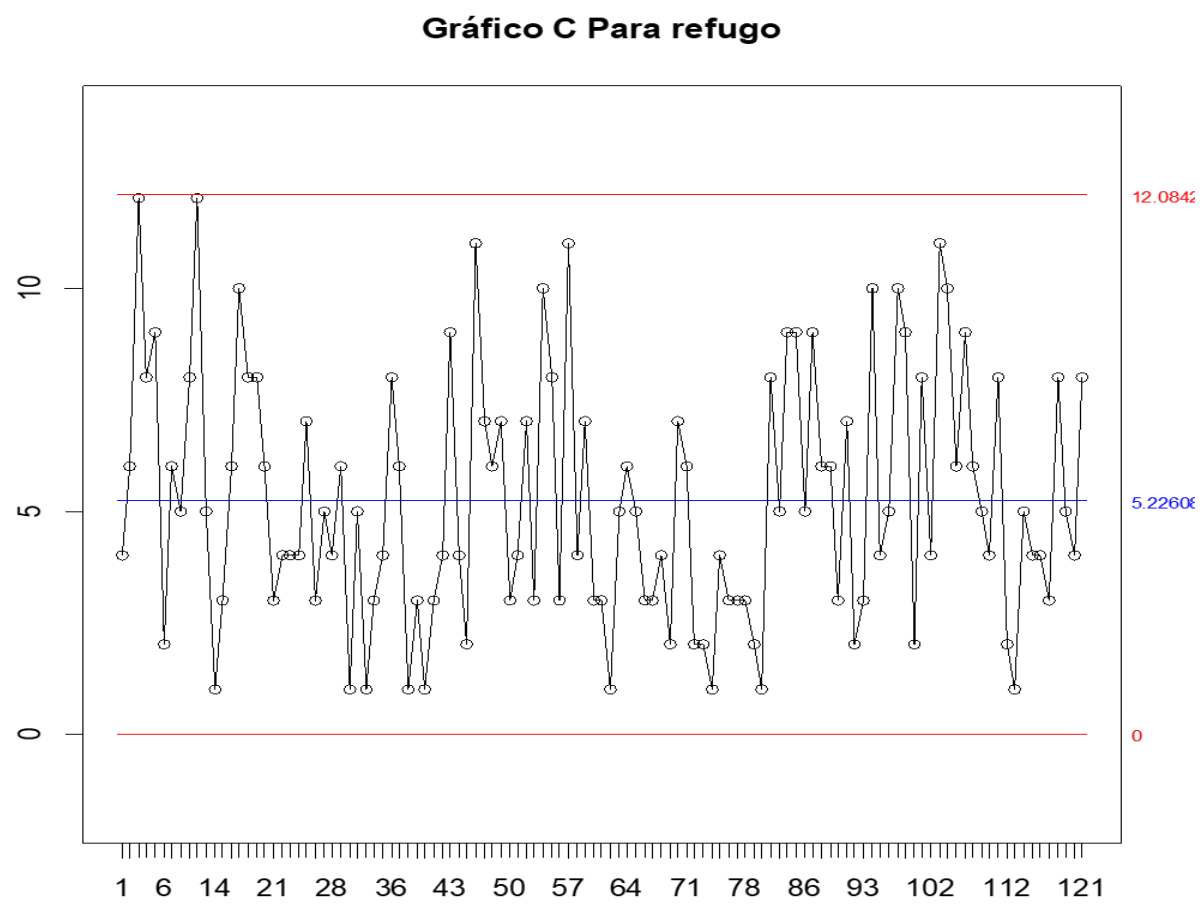

Fonte: Elaborada pelo autor.

Com os resultados apresentados na Figura 4, a equipe segue para quinta etapa de implantação da CEP avaliando em termos quantitativos e econômicos quanto que cada observação está fora da média. Após estabelecer um controle e acompanhar a variabilidade dos eventos a equipe decide utilizar de outra ferramenta de CEP, o gráfico de Pareto.

Montgomery (2019) propõe a utilização de outra ferramenta estatística, o gráfico de Pareto, que conforme contextualizado anteriormente é responsável por identificar quais eventos correspondem ao maior impacto sobre o conjunto de dados. Conforme demonstração gráfica na Figura 5. 


\section{Figura 5 - Gráfico de Pareto.}

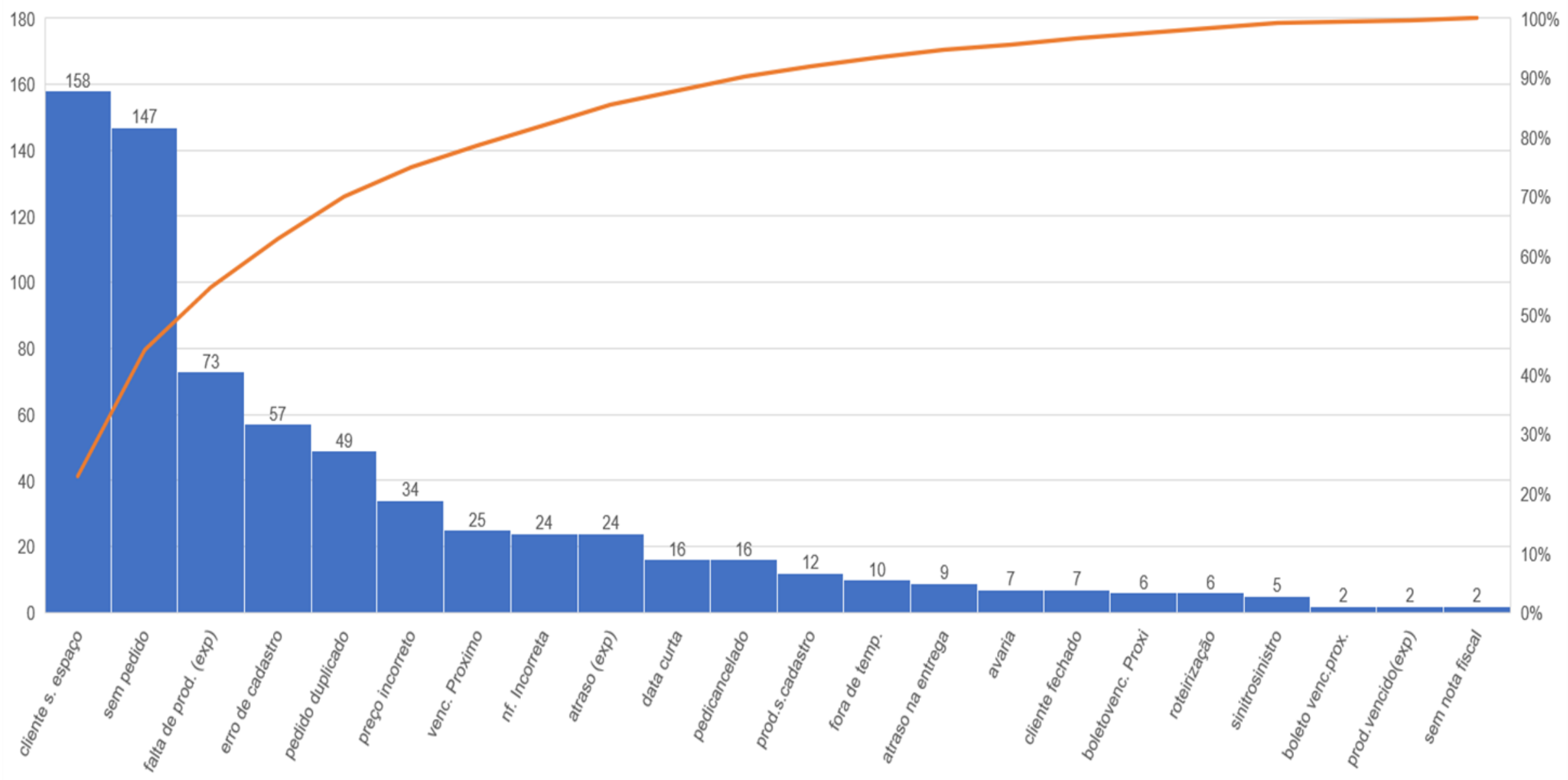

Fonte: Elaborada pelo autor. 
Após a aplicação do Gráfico de Pareto, é possível identificar nas 121 observações, um total de 691 devoluções e 22 variáveis respectivamente representadas. Com os resultados demostrados na Figura 5, a equipe torna-se capaz analisar de forma objetiva cada indicador e sua particularidade, nesta análise a equipe define dividir os resultados apresentados, em dois grupos de causadores: "Grupol-cliente embarcador" e "Grupo 2- operador logístico" conforme demonstração nas Tabelas 1 e 2, abaixo:

Tabela 1- Grupo 1 (Cliente Embarcador).

\begin{tabular}{|l|c|c|c|}
\hline \multicolumn{4}{|c|}{ ANÁLISE DE DEVOLUÇÃO-GRUPO 1 } \\
\hline CAUSADORES & KG DEVOLVIDO & QTD. DEVOLUÇÃO & PORCENTAGEM \\
\hline cliente s. espaço & $24.189,2$ & 158 & $22,87 \%$ \\
\hline sem pedido & $19.180,5$ & 147 & $21,27 \%$ \\
\hline erro de cadastro & $15.969,5$ & 57 & $8,25 \%$ \\
\hline pedido duplicado & $8.429,2$ & 49 & $7,09 \%$ \\
\hline preço incorreto & $1.203,4$ & 34 & $4,92 \%$ \\
\hline venc. Proximo & $2.636,5$ & 25 & $3,62 \%$ \\
\hline nf. Incorreta & $7.706,8$ & 24 & $3,47 \%$ \\
\hline data curta & $1.769,1$ & 16 & $2,32 \%$ \\
\hline pedicancelado & $3.061,1$ & 16 & $2,32 \%$ \\
\hline prod.s.cadastro & $1.333,1$ & 12 & $1,74 \%$ \\
\hline boleto venc. Proxi & 991,0 & 6 & $0,87 \%$ \\
\hline TOTAL & 86.470 & 544 & $78,73 \%$ \\
\hline
\end{tabular}

Fonte: Elaborada pelo autor.

Analisando as Tabelas 1 e 2 é possível identificar que 78,73\% de toda devolução analisada é originalizada do cliente embarcador e $21,23 \%$ é originalizada no operador logístico, este percentual representa um total de $111.905,00$ quilos devolvidos no período analisado 
Tabela 2- Grupo 2 (Operador Logístico).

\begin{tabular}{|l|c|c|c|}
\hline \multicolumn{3}{|c|}{ ANÁLISE DE DEVOLUÇÃO -GRUPO 3 } \\
\hline \multicolumn{1}{|c|}{ CAUSADORES } & KG DEVOLVIDO & QTD. DEVOLUÇÃO & PORCENTAGEM \\
\hline falta de prod. (exp) & $10.511,6$ & 73 & $10,56 \%$ \\
\hline atraso (exp) & $2.237,2$ & 24 & $3,47 \%$ \\
\hline fora de temp. & $1.679,5$ & 10 & $1,45 \%$ \\
\hline atraso na entrega & $1.737,0$ & 9 & $1,30 \%$ \\
\hline avaria & $2.811,4$ & 7 & $1,01 \%$ \\
\hline cliente fechado & 337,6 & 7 & $1,01 \%$ \\
\hline roteirização & 372,8 & 6 & $0,87 \%$ \\
\hline sinitrosinistro & 289,8 & 5 & $0,72 \%$ \\
\hline boleto venc.prox.(exp) & 262,4 & 2 & $0,29 \%$ \\
\hline prod.vencido(exp) & 121,4 & 2 & $0,29 \%$ \\
\hline sem nota fiscal & $5.074,7$ & 2 & $0,29 \%$ \\
\hline TOTAL & 25.435 & $\mathbf{1 4 7}$ & $\mathbf{2 1 , 2 7 \%}$ \\
\hline
\end{tabular}

Fonte: Elaborada pelo autor.

A empresa estudada, define o preço de seus serviços através do peso bruto e quantidade entregas efetivadas, com esta definição a equipe mensura o custo operacional ao realizar um retrabalho, processando as devoluções que retornam para o CD da empresa. Com esta mensuração de custos a equipe inicia um novo brainstorming com objetivo de atacar a raiz das devoluções que representam $85 \%$ do índice de devoluções indicado na Figura 5. Para auxiliar a análise é prescrito na Tabela 3, os causadores que representam $85 \%$ do índice de devolução.

Tabela 3 - Análise de devolução.

\begin{tabular}{|c|l|c|c|c|}
\hline \multicolumn{7}{|c|}{ ANÁLISE DE DEVOLUC̃̃ } \\
\hline GRUPO & CAUSADORES & KG DEVOLVIDO & QTD. DEVOLUÇÃO & PORCENTAGEM \\
\hline 1 & cliente s. espaço & 24.189 & 158 & $22,87 \%$ \\
\hline 1 & sem pedido & 19.181 & 147 & $21,27 \%$ \\
\hline 2 & falta de prod. (exp) & 10.512 & 73 & $10,56 \%$ \\
\hline 1 & erro de cadastro & 15.969 & 57 & $8,25 \%$ \\
\hline 1 & pedido duplicado & 8.429 & 49 & $7,09 \%$ \\
\hline 1 & preço incorreto & 1.203 & 34 & $4,92 \%$ \\
\hline 1 & venc. Proximo & 2.637 & 25 & $3,62 \%$ \\
\hline 1 & nf. Incorreta & 7.707 & 24 & $3,47 \%$ \\
\hline 2 & atraso (exp) & 2.237 & 24 & $3,47 \%$ \\
\hline TOTAL & & 92.064 & 591 & $85,5 \%$ \\
\hline
\end{tabular}

Fonte: Elaborada pelo autor.

Na Tabela 3 a equipe identifica que $71,49 \%$ das devoluções analisadas são representados pelo grupo 1 e $14,4 \%$ são representadas pelo grupo 2 , como auxilio para atacar 
a raiz das anomalias, a equipe decide utilizar outra ferramenta do CEP, o diagrama de Ishikawa. Neste diagrama é possível visualizar na linha central a principal causa "devoluções" e os efeitos que influenciaram para existência da mesma, conforme demonstração da Figura 6. 
Figura 6 - Diagrama de Causa e efeito.

\section{DIAGRAMA DE CAUSA E EFEITO}

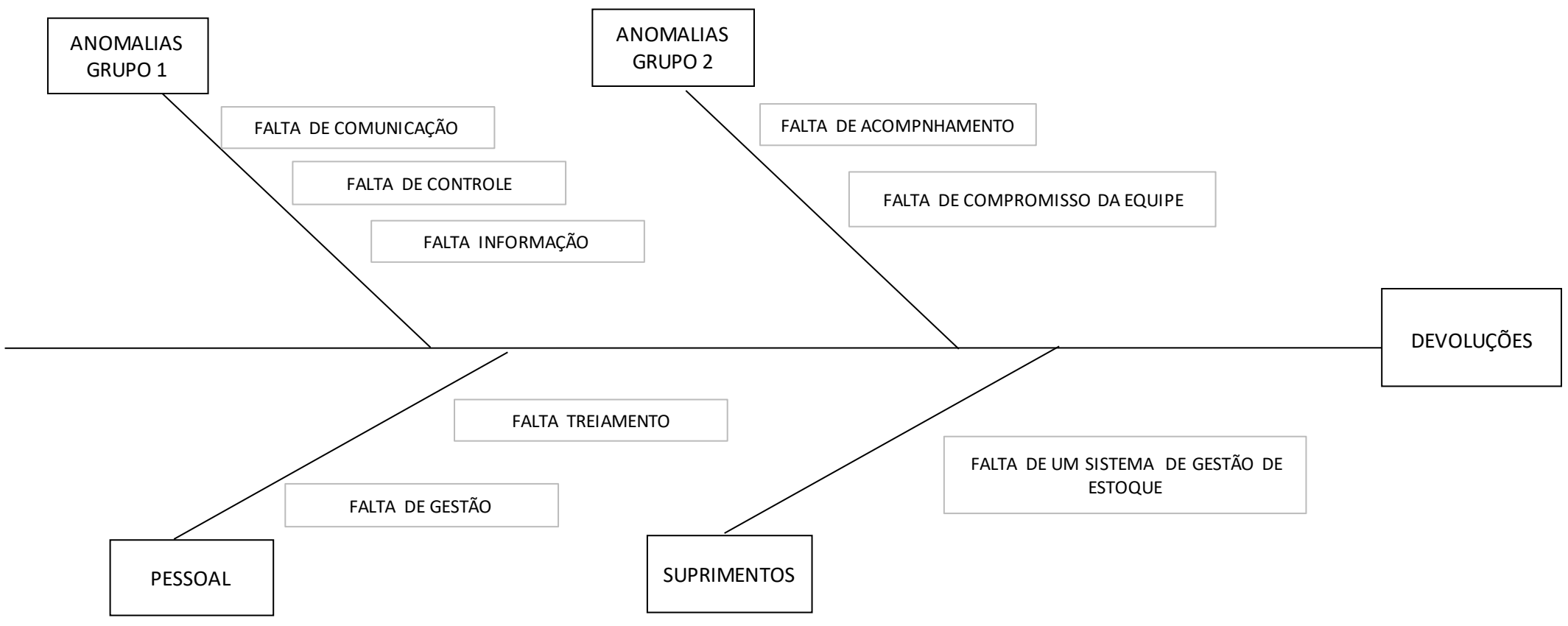

Fonte: Elaborado pelo autor. 
Neste brainstorming, a equipe constatou que há quatro efeitos influenciadores da causa principal "devolução" que são eles "anomalias grupo 1", “anomalias grupo 2 ”, "pessoal " e "suprimentos" estes efeitos são gerados pela falta de comunicação entre o fornecedor e o cliente final, falta de informação do entre o fornecedor e o operador logístico, falta de acompanhamento e controle de processos do operador logístico, falta de gestão do fornecedor e do operador logístico, falta de treinamento do operador logístico e falta de um sistema de gestão de estoque.

Com as causas e os efeitos bem definidos, o gerente junto com a equipe implanta uma ferramenta da qualidade o 5W2H como plano de ação, através desta ferramenta a equipe será capaz de propor melhorias e definir metas operacionais.

O plano de ação definido, tem como principal objetivo reduzir custos operacionais e propor melhorias e ações para inibir o surgimento das devoluções, conforme demonstração da Figura 7. 
Figura 7- Plano de Ação.

\begin{tabular}{|c|c|c|c|c|c|c|}
\hline \multicolumn{7}{|c|}{ 5W2H- PLANO DE AÇÃO DEVOLUÇÕES } \\
\hline ANOMALIA & OQUE & PORQUE & сомо & ONDE & QUANO & QUANTO CUSTA \\
\hline $\begin{array}{l}\text { CLIENTE SEM ESPACOO (ANOMALIA } \\
\text { GRUPO 1) }\end{array}$ & $\begin{array}{l}\text { SOLICIARA AO CLIENTE EMBARCADOR } \\
\text { PARA VISTAR O CLIENTE FINALE } \\
\text { ENTENDER A NECESSIDADE DO } \\
\text { MESMOO }\end{array}$ & $\begin{array}{l}\text { GARANTIR QUE A MERCADORIA NÃO SEJA } \\
\text { DEVOLVIDA PELO CLIENTE FINAL POR } \\
\text { FALTA DE ESPAÇO DO MESMO }\end{array}$ & $\begin{array}{l}\text { ATRAVÉS DE REUNIÖES COM } \\
\text { APRESENTACAOA DE INDICADORES ENTRE } \\
\text { O CLLENTE EMBARCADOR, O OPERADOR } \\
\text { LOGiSTICO E CLIENTE FINAL }\end{array}$ & $\begin{array}{c}\text { EM SALAS DE REUNIÃO DO } \\
\text { FORNECEDOR OU DO CLIENTE FINAL }\end{array}$ & $\begin{array}{l}\text { DEFINICCÃO DO PRAZO APÓS A } \\
\text { APLICAÇÁO DE CEP }\end{array}$ & $\begin{array}{c}\text { TEMPO EM REUNIŌES, NÃO } \\
\text { MENSURÁVES ATÉ A EXECUÇÃO } \\
\text { DA IMPLANTAÇÃO }\end{array}$ \\
\hline $\begin{array}{l}\text { CLIENTE SEM PEDIDO ( ANOMALIA } \\
\text { GRUPO 1) }\end{array}$ & $\begin{array}{l}\text { SOLICTAR CLIENTE EMBARCADOR } \\
\text { ENAMINHAR OPRODUTO PARA SER } \\
\text { DISTRIBUIDO SOMENTE APOS A } \\
\text { CONFIRMACAO DO PEDDO DO } \\
\text { CLIENTE FINAL }\end{array}$ & $\begin{array}{l}\text { O PEDIDO É DEVOLVIDO PELO CLIENTE } \\
\text { FINAL QUE ALEGA NÃO TER PEDIDO O } \\
\text { MESMO AO CLIENTE EMBARCADOR }\end{array}$ & $\begin{array}{l}\text { DISTRIBUIR O PRODUTO SOMENTE APÓS O } \\
\text { CLIENTE EMBARCADOR ENCAMHARA } \\
\text { CONFIRMAÇAOD DO PEDDO FETA PELO } \\
\text { CLIENTE FINAL }\end{array}$ & CD-GILLES LOGISTICA & $\begin{array}{l}\text { DEFFINIC̄ÃO DO PRAZO APÓS A } \\
\text { APLICAÇĀO DE CEP }\end{array}$ & $\begin{array}{l}\text { SEM CUSTO PARA O OPERADOR } \\
\text { LOGISTICO }\end{array}$ \\
\hline $\begin{array}{l}\text { FALTA DE PRODUTO (EXP) } \\
\text { (SUPRIMENTOS GRUPO 2) }\end{array}$ & $\begin{array}{l}\text { IMPLANTAR UM SISTEMA DE GESTÃO } \\
\text { DE ESTOQUE (GESTĀO CLICK) }\end{array}$ & $\begin{array}{l}\text { NÃO EXISTE UM CONTROLE DE ESTOQUE } \\
\text { GERANDO MARGENS PARA ERRO }\end{array}$ & $\begin{array}{l}\text { SOLICTAR A DIRETORIA A AUTORZAÇ̄̃O } \\
\text { DE EFETVR UM EMPENHO PARA } \\
\text { AQUISICSACAO DO SISTEMA }\end{array}$ & CD-GILLES LOGISTICA & $\begin{array}{l}\text { DEFINICCÃO DO PRAZO APÓS A } \\
\text { APLICAÇA̧O DE CEP }\end{array}$ & $7.982,00$ \\
\hline $\begin{array}{l}\text { ERRO DE CADASTRO( PESSOAL- } \\
\text { GRUPO 1) }\end{array}$ & $\begin{array}{l}\text { SOLICTAR O CLIENTE EMBARCADOR } \\
\text { PARA TREINAR A EQUIPE COMERCIAL }\end{array}$ & $\begin{array}{l}\text { CLIENTES FINAIS DEVOLVEM A } \\
\text { MERCADORIA POR ESTAR COM } \\
\text { NFFORMAÇÖES ERRAAAS REDIGIDAS NA } \\
\text { NOTA FISCAL }\end{array}$ & $\begin{array}{l}\text { SERÁ APRESENTADO AO CLIENTE } \\
\text { EMBADOR INDICADORES DE } \\
\text { DEOLUCAOAO SOLICIAR AO MESMO } \\
\text { PARA EFETIVAR TREINAMENTO }\end{array}$ & $\begin{array}{l}\text { CD- GILLES LOGISTICA E SALAS DE } \\
\text { REUNIÄO NO CLIENTE EMBARCADOR }\end{array}$ & $\begin{array}{l}\text { DEFFINIC̄ÃO DO PRAZO APÓS A } \\
\text { APLICAÇĀO DE CEP }\end{array}$ & $\begin{array}{c}\text { TEMPO EM REUNIŌES, NÃO } \\
\text { MENSURÁVEIS ATÉ A EXECUÇÃO } \\
\text { DA IMPLANTAÇĀO }\end{array}$ \\
\hline $\begin{array}{l}\text { PEDIDO DUPLICADO ( PESSOAL- } \\
\text { GRUPO 1) }\end{array}$ & $\begin{array}{l}\text { SOLICTAR O CLIENTE EMBARCADOR } \\
\text { PARA TREINAR A EQUIPE COMERCIAL }\end{array}$ & $\begin{array}{l}\text { CLIENTES FINAIS DEVOLVEM A } \\
\text { MERCADORA POR ESTAR EM } \\
\text { DUPLICIDADE DE PEDIDO }\end{array}$ & $\begin{array}{l}\text { SERÁ APRESENTADO AO CLIENTE } \\
\text { EMBADOR NNDICADORES DE } \\
\text { DEVLUCGAOE SOLICIAR AO MESMO } \\
\text { PARA EFETVARAR TREINAMENTO }\end{array}$ & $\begin{array}{l}\text { CD- GILLES LOGISTICA E SALAS DE } \\
\text { REUNIÄO NO CLIENTE EMBARCADOR }\end{array}$ & $\begin{array}{l}\text { DEFINICCÃO DO PRAZO APÓS A } \\
\text { APLICAÇĀO DE CEP }\end{array}$ & $\begin{array}{l}\text { TEMPO EM REUNIÖES, NÃO } \\
\text { MENSURAVEIS ATE A EXECUÇĀO } \\
\text { DA IMPLANTAÇĀAOO }\end{array}$ \\
\hline $\begin{array}{l}\text { PREÇO INCORRETO ( PESSOA- } \\
\text { GRUPO 1) }\end{array}$ & $\begin{array}{l}\text { TRATATIVA COMERCIAL DO CLIENTE } \\
\text { EMBARCADOR }\end{array}$ & NÃO MENSURÁVEL & NÃO MENSURÁVEL & NÃO MENSURÁVEL & NÃO MENSURÁVEL & $\begin{array}{l}\text { SEM CUSTO PARA O OPERADOR } \\
\text { LOGISTICO }\end{array}$ \\
\hline $\begin{array}{l}\text { VENC. DO PRODUTO PRÓXIMO ( } \\
\text { ANOMALIA GRUPO 1) }\end{array}$ & $\begin{array}{l}\text { ORIENTAR O CLIENTE EMBARCADOR } \\
\text { SOBRE AS DATAS DOS PRODUTOS } \\
\text { NO ATO DO RECEBIMENTO. }\end{array}$ & $\begin{array}{l}\text { DNIDO AO TEMPO EM TRANSTIO A } \\
\text { MERCADORIA JÁ CHEGA COM A DATA } \\
\text { CURTA, }\end{array}$ & $\begin{array}{l}\text { TODA MERCADORIA QUE CHAGAR } \\
\text { DEVER'PA SER REPASSADO } \\
\text { IMEDIATAMENTE A DATA DA MESMA PARA } \\
\text { OCLIENTE EMBARCADOR PAR }\end{array}$ & CD-GILLES LOGISTICA & $\begin{array}{l}\text { DEFFINIC̄ÃO DO PRAZO APÓS A } \\
\text { APLICAÇĀO DE CEP }\end{array}$ & $\begin{array}{l}\text { SEM CUSTO PARA O OPERADOR } \\
\text { LOGISTICO }\end{array}$ \\
\hline $\begin{array}{l}\text { NF. INCORRETA ( ANOMALIA GRUPO } \\
1 \text { ) }\end{array}$ & $\begin{array}{l}\text { TRATATIVA COMERCIAL DO CLIENTE } \\
\text { EMBARCADOR }\end{array}$ & NÃO MENSURÁVEL & NÃO MENSURÁVEL & NÃO MENSURÁVEL & NÃO MENSURÁVEL & $\begin{array}{l}\text { SEM CUSTO PARA O OPERADOR } \\
\text { LOGISTICO }\end{array}$ \\
\hline $\begin{array}{l}\text { ATRASO EXPEDIC̄ÃO (PESSOAL- } \\
\text { GRUPO 2) }\end{array}$ & $\begin{array}{l}\text { TREINARA EQUIPE DE GESTÃO DO } \\
\text { SETRR, CONTROLARA EQUIPE } \\
\text { OPEACIONAL E OFERECER } \\
\text { TREINAMENTOS ESPECIFICOS }\end{array}$ & $\begin{array}{l}\text { A GESTÃO NÃO CONTROLA OS } \\
\text { OPERADORES E FALTA TREIMMENTOP } \\
\text { PARA TODA A EQUIPE }\end{array}$ & ATRAVÉS DE CURSOS ESPECÍFICOS & SEST SENAT- CARIACICA-ES & $\begin{array}{l}\text { DEFINICÃ̃O DO PRAZO APÓS A } \\
\text { APLICAÇĀO DE CEP }\end{array}$ & $\begin{array}{l}\text { SEM CUSTO PARA O OPERADOR } \\
\text { LOGISTICO }\end{array}$ \\
\hline
\end{tabular}

Fonte: Elaborado pelo autor. 
Após a aplicação do plano de ação demostrado na Figura 7, o gerente junto com a equipe analisaram que mesmo $71,49 \%$ do índice de devolução pertencer ao cliente embarcador (grupo 1), as ações do (grupo 2) impactam diretamente para reduzir o índice de devolução, através de reuniões entre o operador logístico e cliente embarcador, investimento de treinamento para equipe, investimento de sistema de gestão de estoque e relacionamento interpessoal entre o cliente final, cliente embarcador e operador logístico e aplicação de CEP, há uma possibilidade de reduzir $85 \%$ das devoluções apresentadas neste estudo além de reduzir o custo operacional.

\section{Conclusão}

Este estudo propôs uma pré-implantação de CEP, com objetivo de analisar as devoluções na fase de distribuição da empresa estudada, descrevendo suas respectivas representatividades, tratamentos específicos por devolução e propondo melhorias baseadas nos resultados das ferramentas da qualidade utilizadas.

Utilizando como auxílio o gráfico de controle por atributos tipo c, foi possível acompanhar a variabilidade das devoluções de uma transportadora no período de 121 dias e constatar que o processo estava fora de controle, um total de 691 devoluções foram geradas no período analisado. Como forma de análise foi utilizado pela equipe da empresa outra ferramenta CEP, o gráfico de Pareto, Figura 5.

Através do gráfico de Pareto a equipe foi capaz de analisar e responder uma pergunta deste estudo: Quais devoluções têm a maior representatividade dentro dos dados analisados? Identificando que $78,73 \%$ de toda devolução analisada está é originalizada do cliente embarcador e $21,23 \%$ é originalizada no operador logístico, este percentual representa um total de 111.905,00 quilos devolvidos, essa logística reversa gera custos operacionais para o operador logístico.

Uma sessão brainstorming entre o gerente e a equipe foi proposta neste estudo e foi identificado que mesmo $78,73 \%$ das devoluções sendo causadas pelo cliente embarcador, o custo do um retrabalho em processar estas devoluções era assumido pelo operador logístico, ressaltando que a empresa estudada estipula o valor de seus serviços para processar os produtos para serem entregues não para serem devolvidos, nesta mesma sessão a equipe decidiu atacar a raiz das devoluções com objetivo de reduzir em $85 \%$ o índice de devoluções indicado na Figura 5. Após a definição da meta a equipe conseguiu responder outra pergunta 
deste estudo: Quais as tratativas necessárias para cada tipo de devolução? Aplicando o diagrama de Ishikawa, para auxiliar a classificar as causas e os efeitos que influenciavam para o surgimento das devoluções, nesta etapa a equipe também respondeu a outra pergunta deste artigo: Quais soluções propor para estabilizar as anomalias no processo? Aplicando em conjunto com o diagrama de Ishikawa a fermenta $5 \mathrm{~W} 2 \mathrm{H}$ como plano de ação onde foi atacado cada causador de devolução e proposto melhorias para estas causas.

Após a aplicação deste plano de ação, o gerente junto com a equipe constatou que através da ferramenta CEP em conjunto com outras ferramentas da qualidade o processo de devolução pode ser estabilizado e após a aplicação de todas ações demostradas na Figura 7, há uma possibilidade de reduzir de $85 \%$ das devoluções da empresa estudada.

Considerando que o estudo deste caso foi uma investigação empírica de uma transportadora houve limitações na aplicação das de brainstorming, devido a rotina operacional da equipe de aplicação, falta de reuniões entre o cliente embarcador e a empresa estudada e o curto período de análise das devoluções.

Como sugestões para pesquisas futuras, indica-se a aplicação das ferramentas de CEP a longo prazo com objetivo de obter resultados mais consolidados.

Neste contexto este estudo atingiu os objetivos propostos no mesmo e contribuiu para uma possível melhoria no nos resultados processo de devolução da empresa estudada, resultados estes que foram demostrados através de um mecanismo de comunicação visual para toda empresa.

\section{REFERÊNCIAS}

ANDRADE, Jairo José de Oliveira. DE O., e C. DIEHL. Avaliação do processo de fabricação de blocos de concreto através do emprego do controle estatístico de processo. ABEPRO, 4 a 7 de outubro de 2011.

ANDRÉ, M. E. D. A. Estudo de caso em pesquisa e avaliação educacional. Brasília: Liberlivro, 2005.

BAETA, Rodrigo. Conheça os principais erros e desafios de uma gestão de projetos, 2017. Disponível em: https://www.siteware.com.br/projetos/conheca-principais-errosdesafios-de-gestao-de-projetos/ Acessado 08/11/2019.

CAMAROTTO, M. R. Gestão de atacado e varejo. Curitiba: Iesde Brasil SA,2009 comparativo de dois clusters da cidade de São Paulo. Gestão \& Regionalidade, v. 29, n. 85, p. 47-62, 2013. 
CHOPRA, Sunil; MEINDL, Peter. Gerenciamento da Cadeia de Suprimentos: estratégia, planejamento e operação. São Paulo: Prentice Hall, 2003.

COLLIS, Jill; HUSSEY, Roger. Pesquisa em administração: um guia prático para alunos de graduação e pós-graduação. 2. ed. Porto Alegre: Bookman.

Lachman L, Lieberman HÁ, Kanig JL. Teoria e prática na indústria farmacêutica. Lisboa: Fundação Coloutre Gulbekian, 2001. p.1379-88.

LAKATOS, E. M.; MARCONI, M. de A. Fundamentos da metodologia científica. 6. Ed. 7. Reimp. São Paulo: Atlas, 2009.

MARTINS, G. P.; LAUGENI, P. F. Administração da produção. 2. ed. São Paulo: Saraiva, 2005.

MONTGOMERY, Douglas C. Introdução ao controle estatístico da qualidade. Rio de Janeiro. LTC - Livros Técnicos e Científicos Editora S.A, 2019.

PALADINI, E. P. et al. Gestão da Qualidade - Teoria e Casos. 2. Ed. Rio de Janeiro: Elsevier, 2012.

PEINADO, J.; GRAEML, R. A. Administração da produção: operações industriais e de serviços. Curitiba: UnicenP, 2007.

PROBST, Gilbert; RAUB, Steffen; ROMHARDT, Kai. Gestão do conhecimento: os elementos construtivos do sucesso. Porto Alegre: Bookman, 2002.

SELEME, R.; HUMBERTO, S. Controle de qualidade: as ferramentas essenciais Curitiba: InterSaberes, 2012.

TOLEDO, José Carlos de; BORRÁS, Miguel Ángel A.; MERGULHÃO Ricardo Coser; MENDES Glauco H. S. Qualidade. Gestão e Métodos. Rio de Janeiro: LTC, 2014.

WANKE, P. Impactos da Sofisticação Logística de Empresas Industriais nas Motivações para Terceirização. Revista Gestão \& Produção, Nov, 2004.

YIN, R. K. Estudo de caso: planejamento e métodos. Tradução: Daniel Grassi. 2. ed. Porto Alegre: Brookman, 2001. 212 p. 